\title{
A non-local model of thermal energy transport: The fractional temperature equation
}

\author{
Maria Stella Mongioví ${ }^{a}$, Massimiliano Zingales ${ }^{\mathrm{b}, *}$ \\ a Dipartimento di Energia, Ingegneria dell'informazione e modelli matematici (DEIM), Viale delle Scienze ed.9, 90128 Palermo, Italy \\ ${ }^{\mathrm{b}}$ Dipartimento di Ingegneria Civile, Ambientale, Aerospaziale, dei Materiali (DICAM), Viale delle Scienze ed.8, 90128 Palermo, Italy
}

\section{A R T I C L E I N F O}

\section{Article history:}

Received 5 June 2013

Received in revised form 21 June 2013

Accepted 10 July 2013

Available online 16 September 2013

\section{Keywords:}

Fractional calculus

Long-range heat transport

Generalized entropy

Temperature field

Non-local thermal energy transport

\begin{abstract}
A B S T R A C T
Non-local models of thermal energy transport have been used in recent physics and engineering applications to describe several "small-scale" and/or high frequency thermodynamic processes as shown in several engineering and physics applications. The aim of this study is to extend a recently proposed fractional-order thermodynamics ([5]), where the thermal energy transfer is due to two phenomena: A short-range heat flux ruled by a local transport equation; a long-range thermal energy transfer that represents a ballistic effects among thermal energy propagators. Long-range thermal energy transfer accounts for small-scale effects that are assumed proportional to the product of the interacting masses, to a distance-decaying function, as well as to their relative temperature. In this paper the thermodynamic consistency of the model is investigated obtaining some restrictions on the functional class of the distance decaying function that rules the strength of the long-range thermal energy transfer. As the distance-decaying function is assumed in the form of a power-law decay a novel temperature equation involving multidimensional spatial Marchaud $\alpha$-order derivatives $(0 \leqslant \alpha \leqslant 1)$ of the temperature field in the body is obtained. Some analytical and numerical solutions of the fractional-order temperature equation have been provided in the paper to show the capabilities of the proposed model and the influence of model parameters.
\end{abstract}

(c) 2013 Elsevier Ltd. All rights reserved.

\section{Introduction}

In recent years the use of new complex and very performing materials has became common in engineering and physical applications as well as in the border sciences as biophysics and biomechanics. In all these cases the presence of a complex, often multi-scale objects are involved and the need for accurate temperature description is no more fulfilled by classical thermodynamics. In this regard, several authors begun to use non-local version of the transport equations in terms of integral models [1,2] or, alternatively, by the introduction of gradients of the state variables in the transport equations. These approaches are very similar to the integral and gradient models of non-local elasticity [3]. Indeed the main drawbacks of these models relies in the lack of physical picture associated to long-range thermal energy transport showing, consequently, severe drawbacks in the positions of Neumann boundary conditions for all the applications where the Kapitza effect is significant affecting the temperature distribution due to phonon propagations away from thermal sources [4].

\footnotetext{
* Corresponding author. Tel.: +3909123896763.

E-mail addresses: mstella.mongiovi@unipa.it (M.S. Mongioví), massimiliano. zingales@unipa.it (M. Zingales).
}

Very recently a new, non-local model of thermal energy transport in rigid bodies with a precise physical description of the thermal energy exchange has been proposed in the context of fractional-order non-local thermodynamics [5]. The basic idea beyond the long-range thermal energy transport is that the scale of heat propagation among non-adjacent locations of the solid is different of order of magnitudes by the thermal energy exchange among adjacent locations of the body. The long-range transport among non-adjacent location of the body is assumed proportional to the relative temperatures among the exchanging portions of the body, to the product of their interacting masses and to a proper distance-decaying function that accounts for the strength of thermal exchanges among locations $\mathbf{x}$ and $\mathbf{y}$. As a power-law with real exponent is chosen, it has been proved that, in 1D case, the temperature equation involves the so-called Marchaud fractional derivative of order $\alpha \in[0,1]$.

Integro-differential operators, representing real order derivatives and integrals, have been introduced more and more often in several contexts of science and engineering [6] since they are capable to interpolate among the well-known integer-order operators of classical differential calculus proved to be a very powerful tool to study temporal and spatial evolutions of complex systems close to critical points. In this context several applications to polymers, 
gels, foams and glassy materials have been provided yet at the beginning of the nineties [7-9] whereas wider use of fractional differential calculus in other fields of physics and mechanics may be found by the end of the nineties of the last century [10-13]. Beside the interpolation features of fractional-order operators another interesting capability of real-order derivatives is related to the long-tails of operators. This feature is widely used in the context of viscoelasticity to describe the not too slow but not too fast either behavior of relaxation test on polymeric materials [14] and biological tissues [15]. The long-memory features of fractional operators has been set, in a spatial context, to formulate a non-local stressstrain relation [16] that is related to integral model of non-local elasticity [17] as it has been recently shown [18].

The use of power-law kernels in the integral model of non-local thermodynamics yields a fractional model of thermal energy transfer in rigid as well as in elastic bodies [19-21] often used to describe thermal energy transfer in nano-systems[22,23]. In such cases fractional models aim to capture, also, the well-known second-sound effect [24-27] involving also the presence of fractional time derivatives of the temperature field $[28,29]$.

In this paper the authors aim to introduce a general framework of non-local thermal energy transport assessing the thermodynamic restriction of the distance-decaying function class for heterogeneous bodies. The choice of the distance decaying function in the class of power-laws, for homogeneous rigid body, yields the temperature equation in terms of the multidimensional Marchaud fractional derivatives assuming that, also the presence of local transport equation is significative in the thermal phenomenon. Analytical solutions as well as numerical results about temperature distributions are conveniently reported in the paper.

\section{A physical picture of long-range thermal energy transfer in rigid bodies}

The presence of non-local thermal energy transport in several physical applications at small scale is usually due to the Kapitza phonon transport effect in close vicinity of the boundaries. A possible perspective of such an effect may be faced in the context of long-range thermal energy transport as proposed in [5] in the context of 1D fractional-order calculus. In this paper we consider an isotropic solid body at rest encapsulated in a subset $V \subset \mathbb{R}^{3}$. We define $u=u(\mathbf{x} ; t)$ as the specific internal energy at location $\mathbf{x} \in \mathbb{R}^{3}$ of the body and time $t$ and $\varrho=\varrho(\mathbf{x})$ the body density at location $\mathbf{x}$ that is not time-dependent as we assume a closed thermodynamical system. The absolute temperature of the body is denoted as $T(\mathbf{x}, t)$, whereas $C_{V}(\mathbf{x})$ is the constant volume specific heat. In the context of non-local thermodynamics we will suppose that, at location $\mathbf{x}=\left(x_{1}, x_{2}, x_{3}\right)$ of the body, the internal energy density of the body may be expressed as $\varrho(\mathbf{x}) u(\mathbf{x}, t)=\varrho(\mathbf{x})\left[u_{l}(\mathbf{x}, t)+u_{n l}(\mathbf{x}, t)\right]$, where $u_{l}(\mathbf{x}, t), u_{n l}(\mathbf{x}, t)$ are the specific local and the long-range residual to the internal energy at location $\mathbf{x}$. The rate of variation of the internal energy, in presence of an energy source $\varrho(\mathbf{x}) r(\mathbf{x})$ reads:

$\frac{\partial(\varrho(\mathbf{x}) u)}{\partial t}=\frac{\partial\left(\varrho(\mathbf{x}) u_{l}\right)}{\partial t}+\frac{\partial\left(\varrho(\mathbf{x}) u_{n l}\right)}{\partial t}+\varrho(\mathbf{x}) r(\mathbf{x})$

The balance relation reported in Eq. (1) represents the non-local first principle of thermodynamics and it involves the following different contributions that are related to the local and non-local thermal energy transfer, respectively, as:

1. The classical thermal energy flux among adjacent locations, that it is related to the divergence $\nabla \cdot \mathbf{q}$ of the heat flux density vector $\mathbf{q}$ and that may be expressed for example, in terms of transport equation provided by the Fourier law as in Classical
Irreversible Thermodynamics (CIT), or instead that can be supposed an independent variable, as in Extended Irreversible Thermodynamics (EIT) [30,31].

2. A non-local energy transfer, due to the contribution of all the other elements $\mathbf{y} \in \mathbb{R}^{3}$ of the body; This latter contribution represents the non-local residual energy that is stored at location $\mathbf{x}$ and it is due to the phonon-phonon transport appearing at smaller (quantic) scales. We will assume that this latter contribution is proportional to the mass densities of the interacting elements at the locations $\mathbf{x}$ and $\mathbf{y}$ as:

$$
\mathcal{P}^{(n l)}(\mathbf{x}, \mathbf{y} ; t)=\chi^{(n l)}(\mathbf{x}, \mathbf{y} ; t) \rho(\mathbf{x}) \rho(\mathbf{y}) d V_{\mathbf{x}} d V_{\mathbf{y}}
$$

where $\chi^{(n l)}(\mathbf{x}, y ; t) \rho(\mathbf{y}) d V_{\mathbf{y}}$ is the long-range specific energy per unit time transferred at locations $\mathbf{x}$ by the element at the location $\mathbf{y}$ and $\rho$ is the mass density that is time-independent.

The balance law of the energy in presence of an energy source $\rho(\mathbf{x}) r(\mathbf{x}, t)$ is provided in the form given by:

$\frac{\partial u(\mathbf{x}, t)}{\partial t}=-\frac{\nabla \cdot \mathbf{q}(\mathbf{x}, t)}{\rho(\mathbf{x})}+\int_{V_{\mathbf{y}}} \chi^{(n l)}(\mathbf{x}, \mathbf{y} ; t) \rho(\mathbf{y}) d V_{\mathbf{y}}+r(\mathbf{x}, t)$

where the integral at the right-hand side is the non-local heat transfer contribute that is due to the interaction between the particle located at position $\mathbf{x}$ and all the other particles of the body [26]. The long-range contribution field, namely function $\chi_{n l}(\mathbf{x}, \mathbf{y} ; t)$, it is assumed, in the model, isotropic and proportional to the relative temperatures among locations $\mathbf{x}$ and $\mathbf{y}$, namely $\triangle_{y} T(x, t)=T(\mathbf{y}, t)-T(\mathbf{x}, t)$, in the form:

$$
\begin{aligned}
\chi^{(n l)}(\mathbf{x}, \mathbf{y} ; t) & =\kappa_{\alpha} g(\|\mathbf{x}-\mathbf{y}\|)[T(\mathbf{y}, t)-T(\mathbf{x}, t)] \\
& =\kappa_{\alpha} g(\|\mathbf{x}-\mathbf{y}\|) \triangle_{y} T(\mathbf{x}, t)
\end{aligned}
$$

with $\kappa_{\alpha}$ a material dependent proportionality coefficient, function $g(\|\mathbf{x}-\mathbf{y}\|)$ is a spatially-decaying function that describes the influence of thermal sources at location $\mathbf{y}$ on the energy density transferred at location $\mathbf{x}$ and $\|\mathbf{x}-\mathbf{y}\|$ is the Euclidean Distance among location $\mathbf{x}$ and $\mathbf{y}$.

In some papers a spatial power-law decay for the decaying function $g(\|\mathbf{x}-\mathbf{y}\|)$ is used. This assumption yields a fractional-order heat conduction equation involving Marchaud-type fractional operators in 1D unbounded domains whereas in 1D bounded domains involves, only, integral contributions to the Marchaud fractional derivatives [5]. This latter consideration yields that the fractional model of long-range heat transport proposed in for $1 \mathrm{D}$ domain is very attractive to handle non-homogeneous Dirichlet boundary conditions on the temperature field since non-divergent algebraic terms at the borders are involved as well as in the position of the Neumann boundary conditions. Similar considerations may also be provided for the thermal energy transfer in three dimensional bodies since the overall residual of the long-range thermal energy transport across the border of the solid vanishes as:

$\int_{V_{\mathbf{x}}} \int_{V_{\mathbf{y}}} \chi^{(n l)}(\mathbf{x}, \mathbf{y} ; t) \rho(\mathbf{y}) \rho(\mathbf{x}) d V_{\mathbf{y}} d V_{\mathbf{x}}=0$

for any specific class of the specific long-range heat transport in the form provided by Eq. (4), yielding that the Neumann boundary conditions on the temperature field involves the local contribution to the heat flux. In this paper, the authors aim to extend the longrange thermal energy transfer model proposed in 1D domain [5] to a more general functional class describing the decay of transport and assessing the thermodynamic restriction that must be fulfilled by the decaying function used to capture the non-local effects.

The temperature equation corresponding to the use of a Fourier transport equation $\mathbf{q}(\mathbf{x}, t)=-\lambda \nabla T(\mathbf{x}, t)$ for the local contribution yields an integro-differential temperature field equation as: 


$$
\begin{aligned}
\dot{T}(\mathbf{x}, t)= & \frac{\lambda \nabla^{2} T(\mathbf{x}, t)}{C_{V} \rho(\mathbf{x})}+\frac{\kappa_{\alpha}}{C_{V}} \int_{V_{\mathbf{y}}} g(\|\mathbf{x}-\mathbf{y}\|) \triangle_{y} T(\mathbf{x}, t) \rho(\mathbf{y}) d V_{\mathbf{y}} \\
& +\frac{r(\mathbf{x}, t)}{C_{V}}
\end{aligned}
$$

where we assumed that the state function represented by the internal energy density depends, only, by the absolute temperature as in classical irreversible thermodynamics $u=u(T)$ and the chain rule of differentiation has been used as $\dot{u}(T)=C_{V} \dot{T}$. The non-local transfer that we reported in Eq. (3) relies on the assumption of local thermodynamic equilibrium conditions. Indeed the use of Fourier model to represent local thermal energy fluxes, involves instantaneous propagation of thermal disturbances in the heat conductor without any second-sound effect. Such an assumption is appropriate for usual engineering applications but, as thermal energy transport involves high frequency processes with oscillation periods comparable with the relaxation times of phonons carrying thermal energy transfer, then an appropriate extension of Fourier transport equations must be accounted for (see e.g. [32]). In this case we will consider a first-order relaxation of thermal energy transfer by means of the Cattaneo model for local thermal energy exchange [33] that reads:

$$
\tau \dot{\mathbf{q}}(\mathbf{x}, t)+\mathbf{q}(\mathbf{x}, t)=-\lambda \nabla \mathrm{T}(\mathbf{x}, t)
$$

that involves, in presence of long-range thermal energy transfer, a hyperbolic temperature equation that allows the propagation of temperature waves. In presence of long-range heat transport, such a temperature equation is obtained as we introduce the gradient operator $\nabla[\bullet]$ to both sides of Eq. (7), yielding:

$\tau \frac{\partial}{\partial t}[\nabla \cdot \mathbf{q}(\mathbf{x}, t)]+[\nabla \cdot \mathbf{q}(\mathbf{x}, t)]=-\lambda \nabla^{2} \mathrm{~T}(\mathbf{x}, t)$

The temperature equation will be obtained replacing the expression in Eq. (8) into the energy balance equation in Eq. (3), accounting for $\dot{u}(T)=C_{V} \dot{T}$. The field equation describing the temperature distribution in this case is a hyperbolic-type, integro-differential equation expressed as:

$$
\begin{aligned}
& (\tau \ddot{T}(\mathbf{x}, t)+\dot{T}(\mathbf{x}, t))-\frac{\kappa_{\alpha}}{C_{V}} \int_{V_{\mathbf{y}}} g(\|\mathbf{x}-\mathbf{y}\|)\left[\tau \triangle_{y} \dot{T}(\mathbf{x}, t)-\triangle_{y} T(\mathbf{x}, t)\right] \rho(\mathbf{y}) d V_{\mathbf{y}} \\
& =\lambda \frac{\nabla^{2} \mathrm{~T}(\mathbf{x}, t)}{\rho(\mathbf{x}) \mathrm{C}_{V}}+r(\mathbf{x}, t)+\dot{r}(\mathbf{x}, t)
\end{aligned}
$$

The temperature equations for the Fourier and Cattaneo model of local thermal energy transport, respectively, in Eqs. (6) and (9) must be supplemented by the Dirichlet and Neumann boundary conditions as well as by the initial temperature distribution (Fourier) with its first-order time derivative (Cattaneo). The boundary conditions do not involve the presence of the residual long-range thermal energy transport since the outgoing net flux of long-range thermal energy outside the body domain vanishes for the symmetry of the decaying function in the integral residual as shown in Eq. (5).

\section{Thermodynamic consistency of the long-range thermal energy transfer model}

In this section the thermodynamic consistency of the generalized model of long-range thermal energy transport presented in previous section will be assessed in the context of the second principle of thermodynamics either for the Fourier local model as well as for the Cattaneo local version of thermal energy transfer. The fulfillment of the second principle provides restrictions on the functional class of the distance-decaying function that may be used to describe the long-range thermal energy exchange in the body domain.

\subsection{The Fourier model of local energy transport}

The compatibility of the proposed model must be framed in the context of the second principle of thermodynamic to validate the physical picture of the fractional-order, long-range, thermal energy transfer in rigid bodies. To this aim we consider the entropy state function of the whole body, namely, $S(t)$ that must obey to Clauses-Planck inequality for a thermodynamical system evolving from state $A \rightarrow B$, occurring, for any location $\mathbf{x}$, at time instants $t_{A}$ and $t_{B}$, respectively:

$\triangle S_{A B}(\mathbf{x})=S\left(\mathbf{x}, t_{B}\right)-S\left(\mathbf{x}, t_{A}\right)=\int_{t_{A}}^{t_{B}} \rho \dot{s}(\mathbf{x}, t) d t \geqslant \int_{t_{A}}^{t_{B}} \frac{d Q(\mathbf{x}, t)}{T(\mathbf{x}, t)}$

where, we introduced the specific entropy function rate $\dot{s}(\mathbf{x}, t)$ and $d Q$ is the thermal energy increment of the body. Recalling that, in a rigid body, $s=s(u)$ and that $\frac{\partial s}{\partial u}=\frac{1}{T}$, making use of the energy balance equation in Eq. (3) the right hand side of Eq. (10) may be written in the form:

$\dot{s}(\mathbf{x}, t) \geqslant-\frac{\nabla \cdot \mathbf{q}(\mathbf{x}, t)}{\rho(\mathbf{x}) T(\mathbf{x}, t)}+\frac{r(\mathbf{x}, t)}{T(\mathbf{x}, t)}+\frac{1}{T(\mathbf{x}, t)} \int_{V_{\mathbf{y}}} \rho(\mathbf{y}) \chi^{(n l)}(\mathbf{x}, \mathbf{y}, t) d V_{\mathbf{y}}$

The observation of Eq. (11) for the specific entropy rate increment shows that an additional contribution at the right-hand side is obtained with respect to the classical expression of the ClausisPlanck inequality. Eq. (11) may be recast in a more convenient form, introducing the specific entropy rate production $\sigma^{(s)}(\mathbf{x}, t) \geqslant 0$ for any thermodynamical process and omitting the dependence on the time variable for shorten notations, yielding

$$
\begin{aligned}
& \dot{s}(\mathbf{x})+\frac{\nabla \cdot \mathbf{q}(\mathbf{x})}{\rho(\mathbf{x}) T(\mathbf{x})}-\frac{r(\mathbf{x})}{T(\mathbf{x})}-\frac{1}{T(\mathbf{x})} \int_{V_{\mathbf{y}}} \rho(\mathbf{y}) \chi^{(n l)}(\mathbf{x}, \mathbf{y}) d V_{\mathbf{y}}=\sigma^{(s)}(\mathbf{x}) \\
& \quad \geqslant 0
\end{aligned}
$$

On the other hand the entropy rate may be expressed at location $\mathbf{x}$ in the form of a balance among the incoming and the out-coming entropy flux in the unitary time so that:

$\dot{s}(\mathbf{x})=-\frac{\nabla \cdot J_{l}^{(s)}(\mathbf{x})}{\rho(\mathbf{x})}+\int_{V_{\mathbf{y}}} \boldsymbol{J}_{n l}^{(s)}(\mathbf{x}, \mathbf{y}) d V_{\mathbf{y}}+\frac{r(\mathbf{x})}{T(\mathbf{x})}+\sigma^{(s)}(\mathbf{x})$

where we introduced the local and non-local long-range entropy transfer, respectively, $\boldsymbol{J}_{l}^{(s)}(\mathbf{x})$ and $J_{n l}^{(s)}(\mathbf{x}, \mathbf{y})$ that are related to the local and long-range thermal energy transfer, respectively, and contributes, both, to the evolution of the entropy rate at location $\mathbf{x}$. In Eq. (13) and in the following derivations we will omit the explicit dependence on time variable, unless explicitly declared, for shortness' sake.

Eq. (13) may be substituted into Eq. (12) to yield the inequality:

$-\frac{\nabla \cdot \mathbf{q}(\mathbf{x})}{\rho(\mathbf{x}) T(\mathbf{x})}+\frac{1}{T(\mathbf{x})} \int_{V_{\mathbf{y}}} \rho(\mathbf{y}) \chi^{(n l)}(\mathbf{x}, \mathbf{y}) d V_{\mathbf{y}}+\frac{\nabla \cdot J_{l}^{(s)}(\mathbf{x})}{\rho(\mathbf{x})}-\int_{V_{\mathbf{y}}} \boldsymbol{J}_{n l}^{(s)}(\mathbf{x}, \mathbf{y}) d V_{\mathbf{y}} \geqslant 0$

In this context the entropy flux is assumed to be a function of state of the local contribution to the internal energy rate $\boldsymbol{J}_{l}^{(s)}(\mathbf{x})=$ $\varphi_{l}\left(u_{l}\right) \mathbf{q}_{l}(\mathbf{x})$ and, by similar considerations we will assume that the long-range entropy transfer is provided as $J_{n l}^{(s)}(\mathbf{x}, \mathbf{y})=$ $\varphi_{n l}\left(u_{n l}\right) \varrho(\mathbf{y}) \varrho(\mathbf{x}) \chi^{(n l)}(\mathbf{x}, \mathbf{y})$. Therefore, the expression reported in Eq. (14) reads:

$$
\begin{aligned}
& \left(\varphi_{l}\left(u_{l}\right)-\frac{1}{T(\mathbf{x})}\right) \nabla \cdot \mathbf{q}(\mathbf{x})+\mathbf{q}(\mathbf{x}) \cdot \nabla\left(\varphi_{l}\left(u_{l}\right)\right) \\
& -\int_{V_{\mathbf{y}}}\left(\varphi_{n l}\left(u_{n l}\right)-\frac{\varrho^{2} \chi^{(n l)}(\mathbf{x}, \mathbf{y})}{T(\mathbf{x})}\right) d V_{\mathbf{y}} \geqslant 0
\end{aligned}
$$

The inequality restriction in Eq. (15) leads to the conclusion that the linear term involving the thermal energy flux $\mathbf{q}(\mathbf{x})$ must vanish yielding the relation: 
$\varphi_{l}\left(u_{l}\right)=\frac{1}{T(\mathbf{x})}$

that, upon substitution into Eq. (15) it yields:

$\frac{\mathbf{q}(\mathbf{x})}{T^{2}(\mathbf{x})} \cdot \nabla(T(\mathbf{x}))+\int_{V_{\mathbf{y}}}\left(\varphi_{n l}\left(u_{n l}\right)-\frac{1}{T(\mathbf{x})}\right) \varrho^{2} \chi^{(n l)}(\mathbf{x}, \mathbf{y}) d V_{\mathbf{y}} \leqslant 0$

The inequality in Eq. (17) for the entropy production may be fulfilled if, for the first term, we assume a linear force-flux relation as:

$\mathbf{q}(\mathbf{x})=-\lambda \nabla(T(\mathbf{x}))$

with $\lambda \geqslant 0$ for the local thermal energy flux, that corresponds to Fourier relation, whereas, the second term at right hand side must involve the inverse of a temperature field $1 / T$ for dimensionality sake. As we assume that the long-range entropy flux function $\varphi_{n l}(-$ $\left.u_{n l}\right)=1 / T(\mathbf{y})$ since $J_{n l}^{(s)}(\mathbf{x}, \mathbf{y})$ represents the entropy flux at location $\mathbf{x}$ exerted by a thermal source at location $\mathbf{y}$, then the integral term must satisfy the inequality:

$\int_{V_{\mathbf{y}}}\left(\frac{T(\mathbf{x})-T(\mathbf{y})}{T(\mathbf{x}) T(\mathbf{y})}\right) \chi^{(n l)}(\mathbf{x}, \mathbf{y}) d V_{\mathbf{y}} \leqslant 0$

that is satisfied assuming for the long-range contribution the form reported in Eq. (4) with kernel $g(\|\mathbf{x}-\mathbf{y}\|) \geqslant 0 \forall \mathbf{x}, \mathbf{y} \in \mathrm{V}$. Such restrictions for the local and non-local thermal energy exchanges are satisfied with the power-law decaying function $g(\|\mathbf{x}-\mathbf{y}\|)=\frac{1}{d} \frac{1}{\|\mathbf{x}-\mathbf{y}\|^{n+\alpha}}$, with $d>0$, yielding full compatibility in the framework of the second thermodynamic principle of the fractional model proposed in [5].

\subsection{The Cattaneo generalized model of local energy transport}

The introduction, of a first-order time lag in the force-flux relation, as in Eq. (7), requires the introduction of a non-equilibrium entropy model functionally dependent on the local internal energy $u$ as well as on the thermal energy flux $\mathbf{q}$ as $s=s(u, \mathbf{q})$. As a consequence the local entropy flux must show the functional dependence $\mathbf{J}_{l}^{(s)}=\mathbf{J}_{l}^{(s)}(u, \mathbf{q})$ [32]. In this setting, the chain rule of differentiation reads for the non-equilibrium entropy rate to:

$\dot{s}=\dot{s}(u, \mathbf{q})=\left(\frac{\partial s}{\partial u}\right)_{\mathbf{q}} \dot{u}+\left(\frac{\partial s}{\partial \mathbf{q}}\right)_{u} \dot{\mathbf{q}}$

In the following we will assume to deal with an isotropic body so that the functional dependence entropy function and local entropy flux reads $s=s(u, \mathbf{q} \cdot \mathbf{q})=s\left(u, q^{2}\right), \mathbf{J}_{l}^{(s)}=\mathbf{J}_{l}^{(s)}\left(u, \mathbf{q}^{2}\right)$ and $\mathbf{J}_{l}^{(s)}=\varphi(u) \mathbf{q}$. The entropy rate function in Eq. (20) reads:

$\dot{s}=\dot{s}\left(u, q^{2}\right)=\left(\frac{\partial s}{\partial u}\right)_{q^{2}} \dot{u}+\left(\frac{\partial s}{\partial q^{2}}\right)_{u} 2 \mathbf{q} \cdot \dot{\mathbf{q}}$

Substitution of Eq. (21) into the entropy balance equation reported in Eq. (13) yields:

$\left(\frac{\partial s}{\partial u}\right)_{q^{2}} \dot{u}+\left(\frac{\partial s}{\partial q^{2}}\right)_{u} 2 \mathbf{q} \cdot \dot{\mathbf{q}}+\frac{\nabla \cdot J_{l}^{(s)}(\mathbf{x})}{\rho(\mathbf{x})}-\int_{V_{\mathbf{y}}} \frac{J n l^{(s)}(\mathbf{x}, \mathbf{y})}{\rho(\mathbf{x})} d V_{\mathbf{y}}-\frac{r}{T(\mathbf{x})}=\sigma^{(s)}(\mathbf{x})$

Replacing the rate of internal energy, with its counterpart in terms of the local and long-range thermal energy transport in Eq. (4) and accounting for Cattaneo transport equation in Eq. (7), after straightforward manipulations, the following relation is obtained:

$$
\begin{aligned}
& \left(\frac{\partial s}{\partial u}\right)_{q^{2}}\left(-\frac{\nabla \cdot \mathbf{q}}{\rho(\mathbf{x})}+\int_{V_{\mathbf{y}}} \rho(\mathbf{y})\left[\chi^{(n l)}(\mathbf{x}, \mathbf{y})-\frac{J n l^{(s)}(\mathbf{x}, \mathbf{y})}{\rho(\mathbf{x})}\right] d V_{\mathbf{y}}\right) \\
& +\frac{\nabla \cdot J_{l}^{(s)}(\mathbf{x})}{\rho(\mathbf{x})}+\left(\frac{\partial s}{\partial q^{2}}\right)_{u} 2 \mathbf{q} \cdot \dot{\mathbf{q}}-\frac{r}{T(\mathbf{x})}=\sigma^{(s)}(\mathbf{x}, t)
\end{aligned}
$$

The derivative of the entropy function with respect to the internal energy, that is $\left(\frac{\partial s}{\partial u}\right)_{q^{2}}$, represents dimensionally a temperature field that, under the assumption of first-order dependence on the heat flux q, it may be assumed coalescing with the absolute temperature of the body as $\left(\frac{\partial s}{\partial u}\right)_{q^{2}}=\frac{1}{T(\mathbf{x})}$ yielding Eq. (23) in the form:

$$
\begin{aligned}
& -\frac{\nabla \cdot \mathbf{q}}{\rho(\mathbf{x}) T(\mathbf{x})}+\frac{1}{T(\mathbf{x})} \int_{V_{\mathbf{y}}} \rho(\mathbf{y}) \chi^{(n l)}(\mathbf{x}, \mathbf{y})-\frac{J_{n l}^{(s)}(\mathbf{x}, \mathbf{y})}{\rho(\mathbf{x})} d V_{\mathbf{y}}+ \\
& -\left(\frac{\partial s}{\partial q^{2}}\right)_{u} 2 \mathbf{q} \cdot\left(\frac{\lambda}{\tau} \nabla T+\frac{\mathbf{q}}{\tau}\right)+\frac{\nabla \cdot J_{l}^{(s)}(\mathbf{x})}{\rho(\mathbf{x})}=\sigma^{(s)}(\mathbf{x}, t)
\end{aligned}
$$

Right-hand side of Eq. (24), namely entropy production rate, must be positive, so that, after some manipulation, the following inequality reads:

$$
\begin{aligned}
& \left(\varphi_{l}-\frac{1}{T(\mathbf{x})}\right) \nabla \cdot \mathbf{q}-\int_{V_{\mathbf{y}}}\left(J_{n l}^{(s)}(\mathbf{x}, \mathbf{y})-\frac{\rho(\mathbf{x}) \rho(\mathbf{y}) \chi^{(n l)}(\mathbf{x}, \mathbf{y})}{T(\mathbf{x})}\right) d V_{\mathbf{y}}+ \\
& -\frac{2 \rho(\mathbf{x}) \lambda}{\tau}\left(\frac{\partial s}{\partial q^{2}}\right) 2 \mathbf{q} \cdot(\nabla T)-\frac{2 \rho(\mathbf{x})}{\tau}\left(\frac{\partial s}{\partial q^{2}}\right) q^{2}+\left(\nabla \varphi_{l}\right) \cdot \mathbf{q} \geqslant 0
\end{aligned}
$$

where we assumed that $\mathbf{J}_{l}^{(s)}=\varphi_{l}(u) \mathbf{q}$. In order to fulfill the condition in Eq. (25) function $\varphi_{l}(u)=\frac{1}{T(\mathbf{x})}$ so that we are left with the following inequality for the remaining terms:

$$
\begin{aligned}
& \int_{V_{\mathbf{y}}}\left(\frac{J_{n l}^{(s)}(\mathbf{x}, \mathbf{y})}{\rho(\mathbf{x})}-\frac{\rho(\mathbf{y}) \chi^{(n l)}(\mathbf{x}, \mathbf{y})}{T(\mathbf{x})}\right) d V_{\mathbf{y}} \\
& +\left(\frac{2 \lambda}{\tau} \mathbf{q} \cdot(\nabla T)+\frac{2}{\tau} q^{2}\right)\left(\frac{\partial s}{\partial q^{2}}\right)+\frac{\nabla T(\mathbf{x})}{\rho(\mathbf{x}) T(\mathbf{x})^{2}} \cdot \mathbf{q} \leqslant 0
\end{aligned}
$$

that must be fulfilled for any thermodynamic process in the body.

The first term in Eq. (26) is analogous to the integral term represented in Eq. (17) as we assume for the long-range entropy transfer the expression $J_{n l}^{(s)}(\mathbf{x}, \mathbf{y})=\varphi_{n l}(u) \rho(\mathbf{x}) \rho(\mathbf{y}) \chi^{(n l)}(\mathbf{x}, \mathbf{y})$ yielding for the non-local proportionality function $\varphi_{n l}(u)=\frac{1}{T(\mathbf{y})}$ to fulfill Eq. (26) for any thermodynamical process. As a consequence, thermodynamic restrictions on long-range heat fluxes involve considerations similar to those reported in previous section about the positivity of the decaying function $g(\|\mathbf{x}-\boldsymbol{y}\|) \geqslant 0$ and they have not reported for brevity. The second term in Eq. (26) satisfies the inequality sign as:

$$
\left(\frac{2 \rho \lambda}{\tau}\left(\frac{\partial s}{\partial q^{2}}\right)+\frac{1}{T(\mathbf{x})^{2}}\right) \mathbf{q} \cdot(\nabla T)+\frac{2 \rho}{\tau}\left(\frac{\partial s}{\partial q^{2}}\right) q^{2} \leqslant 0
$$

that may be fulfilled only as the first-order derivative of the extended entropy function $s=s\left(u, q^{2}\right)$ reads:

$\left(\frac{\partial s}{\partial q^{2}}\right)=-\frac{\tau}{2 \rho \lambda} \frac{1}{T(\mathbf{x})^{2}}$

that yields a thermodynamic restriction upon the signs of the thermal conductivity $\lambda \geqslant 0$ as in Fourier transport equation that we dealt in previous section.

\section{The fractional model of long-range heat transport in homogeneous bodies}

The choice of the distance-decaying function of long-range thermal energy exchange in the body in the functional class of power-laws is thermodynamically consistent since it yields $g(\|\mathbf{x}-\mathbf{y}\|) \geqslant 0$. In this case the temperature equation contains the multidimensional Marchaud-type fractional-order derivatives that represent a generalization of the well-known integer-order derivatives to real order of differentiation. In this section we will discuss this latter case in detail showing that the proposed model of long-range heat transfer represents the generalization to the 3D case of a previously proposed 1D model of thermal energy transfer. 


\subsection{Brief remarks on multivariable fractional calculus}

In this section the essential features of fractional calculus will be shortly discussed. Let us consider a real-valued, Lebesgue integrable function $f(x), x \in \mathbb{R}$ such that $f(x) \in L^{1}$.

The left and right Riemann-Liouville (RL) fractional-order integrals are defined as:

$\left(I_{+}^{\alpha} f\right)(x)=\frac{1}{\Gamma(\alpha)} \int_{-\infty}^{x} \frac{f(y)}{(x-y)^{\alpha}} d y ; \quad\left(I^{\alpha} f\right)(x)=\frac{1}{\Gamma(\alpha)} \int_{x}^{\infty} \frac{f(y)}{(y-x)^{1-\alpha}} d y$

with $\alpha \in[0,1]$ and $\Gamma(\bullet)$ the well-known Euler-Gamma function.

$$
\begin{aligned}
& \left(D_{+}^{\alpha} f\right)(x)=\frac{1}{\Gamma(1-\alpha)} \frac{d}{d x} \int_{-\infty}^{x} \frac{f(y)}{(x-y)^{1-\alpha}} d y \\
& \left(D_{-}^{\alpha} f\right)(x)=\frac{1}{\Gamma(1-\alpha)} \frac{d}{d x} \int_{x}^{\infty} \frac{f(y)}{(y-x)^{1-\alpha}} d y
\end{aligned}
$$

As we assume that function $f(x) \in C^{1}(\mathbb{R})$ with $C^{1}(\mathbb{R})$ the class of continuous functions with continuous first derivative, then the left and right RL fractional derivatives coalesces with the Marchaud (M) fractional operator that is defined as:

$$
\left(\mathbf{D}_{+}^{\alpha} f\right)(x)=\frac{\alpha}{\Gamma(1-\alpha)} \int_{-\infty}^{x} \frac{f(x)-f(y)}{(x-y)^{1+\alpha}} d y=\left(D_{+}^{\alpha} f\right)(x)
$$

for the left $\mathrm{M}$ fractional derivative, whereas, the right $\mathrm{M}$ fractional derivative is related to the right RL fractional derivative as:

$\left(\mathbf{D}_{-}^{\alpha} f\right)(x)=\frac{\alpha}{\Gamma(1-\alpha)} \int_{x}^{\infty} \frac{f(x)-f(y)}{(y-x)^{1+\alpha}} d y=\left(D_{-}^{\alpha} f\right)(x)$

The definition of RL and M fractional derivatives operating on functions defined on bounded intervals $[a, b] \subset \mathbb{R}$ involves integral terms as well as algebraic contributions as:

$$
\begin{aligned}
& \left(D_{a^{+}}^{\alpha} f\right)(x)=\frac{f(a)}{\Gamma(1-\alpha)(x-a)^{\alpha}}+\frac{1}{\Gamma(1-\alpha)} \int_{a}^{x} \frac{f(y)^{\prime}}{(x-y)^{\alpha}} d \xi \\
& \left(D_{b^{-}}^{\alpha} f\right)(x)=\frac{f(b)}{\Gamma(1-\alpha)(b-x)^{\alpha}}-\frac{1}{\Gamma(1-\alpha)} \int_{x}^{b} \frac{f(y)^{\prime}}{(y-x)^{\alpha}} d \xi
\end{aligned}
$$

where $f(y)^{\prime}=\frac{d f}{d y}$, showing divergence at the boundaries of the considered domains, unless function $f(x) \rightarrow 0$ faster than $x^{\alpha}$ as $x \rightarrow a^{+}$ and $x \rightarrow b^{-}$, respectively.

Similar considerations hold true also for the M fractional operators defined on bounded support, yielding:

$$
\begin{aligned}
& \left(\mathbf{D}_{a^{+}}^{\alpha} f\right)(x)=\frac{\alpha f(x)}{\Gamma(1-\alpha)(x-a)^{\alpha}}+\left(\hat{\mathbf{D}}_{a^{+}}^{\alpha} f\right)(x) \\
& \left(\mathbf{D}_{b^{-}}^{\alpha} f\right)(x)=\frac{\alpha f(x)}{\Gamma(1-\alpha)(b-x)^{\alpha}}-\left(\hat{\mathbf{D}}_{b^{-}}^{\alpha} f\right)(x)
\end{aligned}
$$

where $\left(\hat{\mathbf{D}}_{a^{+}}^{\alpha} f\right)(x)$ and $\left(\hat{\mathbf{D}}_{b^{-}}^{\alpha} f\right)(x)$ are the integral parts of the truncated $\mathrm{M}$ fractional operators defined as:

$$
\begin{aligned}
& \left(\hat{\mathbf{D}}_{a^{\prime}}^{\alpha} f\right)(x)=\frac{\alpha}{\Gamma(1-\alpha)} \int_{a}^{x} \frac{f(x)-f(y)}{(x-y)^{1+\alpha}} d y \\
& \left(\hat{\mathbf{D}}_{b^{-}}^{\alpha} f\right)(x)=\frac{\alpha}{\Gamma(1-\alpha)} \int_{x}^{b} \frac{f(x)-f(y)}{(y-x)^{1+\alpha}} d y
\end{aligned}
$$

Equivalent forms to Eqs. (31) and (32) relative to the M fractional derivatives valid for cases involving $\alpha>1$ may be obtained as we introduce the $l$-order finite differences of function $f(x)$, with $l=\{\alpha\}+1$ and $l>1$ (see e.g. [34]) yielding:

$$
\begin{aligned}
\left(\mathbf{D}_{+}^{\alpha} f\right)(x) & =-\frac{1}{\Gamma(-\alpha) A_{l}(\alpha)} \int_{0}^{\infty} \frac{\left(\Delta_{ \pm \xi}^{l} f\right)(x)}{y^{1+\alpha}} d y \\
& =\frac{1}{\chi(l, \alpha)} \int_{0}^{\infty} \frac{\left(\Delta_{ \pm \xi}^{l} f\right)(x)}{y^{1+\alpha}} d y
\end{aligned}
$$

where we denoted $\{\alpha\}$ the integer part of the real number $\alpha$ and the normalization coefficient $\chi(l, \alpha)=-A_{l}(\alpha) \Gamma(-\alpha)$. The fractional finite difference $\left(\Delta_{ \pm \xi}^{l} f\right)(x)$, that appears in the integral term in Eq. (38) and the normalization factor $A_{l}(\alpha)$ are defined as:

$$
\left(\Delta_{ \pm y}^{l} f\right)(x)=\sum_{k=0}^{l}(-1)^{k}\left(\begin{array}{l}
l \\
k
\end{array}\right) f(x-k y) ; \quad A_{l}(\alpha)=\sum_{k=0}^{l}(-1)^{k-1}\left(\begin{array}{l}
l \\
k
\end{array}\right) k^{\alpha}
$$

with $\alpha \in \mathbb{R}^{+}$. The coefficient $A_{l}(\alpha)$ is identically vanishing for integer values of $\alpha=1,2, \ldots, l-1$ whereas the normalization coefficient $\chi(l, \alpha)$ is unbounded as $\alpha \rightarrow l^{-}$and it is finite as $\alpha \rightarrow l^{+}$.

The definitions of Marchaud fractional derivatives applied to scalar functions of simple scalar variables may be extended to scalar functions of multivariable arguments. This extension became more readable as we introduce the Riesz $(R)$ potential operator of function $f$, dubbed $\left(I^{\alpha} f\right)(x)$ that is defined as:

$$
\begin{aligned}
\left(\mathrm{I}^{\alpha} f\right)(x) & =\frac{1}{2 \cos (\alpha \pi / 2) \Gamma(\alpha)} \int_{-\infty}^{+\infty} \frac{f(y)}{\|x-y\|^{1-\alpha}} d y \\
& =\frac{\left(I_{+}^{\alpha} f\right)(x)+\left(I_{-}^{\alpha} f\right)(x)}{2 \cos (\alpha \pi / 2) \Gamma(\alpha)}
\end{aligned}
$$

with inverse operators, namely the D-Riesz fractional differential operator, describing the inverse operator of the Riesz integral that reads as $0 \leqslant \alpha \leqslant 1$ :

$$
\begin{aligned}
\left(\mathrm{I}^{\alpha} f\right)^{-1}(x) & =\left(\mathrm{D}^{\alpha} f\right)(x)=v(\alpha) \int_{-\infty}^{+\infty} \frac{f(x-y)-f(x)}{\|y\|^{1+\alpha}} d y \\
& =v(\alpha) \Gamma(1-\alpha)\left[\left(\mathbf{D}_{+}^{\alpha} f\right)(x)+\left(\mathbf{D}_{-}^{\alpha} f\right)(x)\right]
\end{aligned}
$$

where $v(\alpha)=[2 \alpha \cos (\alpha \pi / 2) \Gamma(\alpha)]^{-1}$. Mathematical expression reported in Eq. (41) shows that, with the exception of the coefficient $v(\alpha) \Gamma(1-\alpha)$ the inverse Riesz potential operator coincide with the sum of left and right Marchaud fractional derivatives. A different, but equivalent, form of the Riesz fractional operator in Eq. (41) may be written as we introduce the fractional difference operator of order $l$, holding for $\alpha \geqslant 0$ as:

$$
\begin{aligned}
\left(\mathrm{I}^{\alpha} f\right)^{-1}(x) & =\left(\mathrm{D}^{\alpha} f\right)(x) \\
& =\frac{v(\alpha) \Gamma(1-\alpha)}{\alpha A_{l}(\alpha) \Gamma(-\alpha)} \int_{0}^{\infty} \frac{\left(\Delta_{+y}^{l} f\right)(x)+\left(\Delta_{-y}^{l} f\right)(x)}{y^{1+\alpha}} d y
\end{aligned}
$$

The expression in Eq. (42) may be easily generalized to the case of fractional generalization of multivariable functions $f(\mathbf{x})$, with $\mathbf{x} \in \mathbb{R}^{n}$ as the $n$ - fold integral:

$\left(\mathrm{I}^{\alpha} f\right)(\mathbf{x})=\frac{1}{\gamma_{n}(\alpha)} \int_{\mathbb{R}^{n}} \frac{f(\mathbf{y})}{\|\mathbf{x}-\mathbf{y}\|^{n-\alpha}} d \mathbf{y} ; \quad \alpha \neq n, n+2, \ldots$

with the normalization constant $\gamma_{n}(\alpha)$ is defined in [35] (Eqs. (25) and (26)).

The inverse operator $\left(\mathrm{I}^{\alpha} f\right)^{-1}(\mathbf{x})=\left(\mathrm{D}^{\alpha} f\right)(\mathbf{x})$, termed as the multivariable Riesz fractional differential operator, is provided as the $n-$ fold integral:

$\left(\mathrm{I}^{\alpha} f\right)^{-1}(\mathbf{x})=\left(\mathrm{D}^{\alpha} f\right)(\mathbf{x})=\frac{1}{d_{n, l}(\alpha)} \int_{\mathbb{R}^{n}} \frac{\left(\triangle_{\mathbf{y}}^{l} f\right)(\mathbf{x})}{\|\mathbf{y}\|^{n+\alpha}} d \xi$

with $\left(\Delta_{\mathbf{y}}^{l} f\right)(\mathbf{x})$ the centered finite difference that represents the extension to higher-dimensional spaces of Eq. (39) defined as:

$$
\left(\Delta_{\mathbf{y}}^{l} f\right)(\mathbf{x})=\sum_{k=0}^{l}(-1)^{k}\left(\begin{array}{l}
l \\
j
\end{array}\right) f(\mathbf{x}-k \mathbf{y})
$$

and where $d_{n, l}(\alpha)$ is a proper normalization constant, that involves an explicit dependence of the fractional order $\alpha$ that reads: 
$d_{n, l}(\alpha)=\beta_{n}(\alpha) \frac{A_{l}(\alpha)}{\sin (\alpha \pi / 2)} ; \beta_{n}(\alpha)=\frac{\pi^{1+n / 2}}{2^{\alpha} \Gamma(1+\alpha / 2) \Gamma(n+\alpha / 2)}$

As it has been shown for the 1D case, the Riesz fractional differential operator $\left(D^{\alpha} f\right)(\mathbf{x})$ may be expressed as the sum of the left and right fractional operators involving fractional differences and henceforth, as in Eq. (44), it may be expressed in terms of the Marchaud fractional derivatives in half-spaces, defined as:

$$
\begin{aligned}
\left(\mathrm{D}^{\alpha} f\right)(\mathbf{x}) & =\frac{\chi_{\bar{l}}^{-}(\bar{\alpha})}{d_{n, \bar{l}}(\bar{\alpha})} \frac{1}{\chi_{\bar{l}}(\bar{\alpha})}\left[\int_{\mathbb{R}_{+}^{n}} \frac{\left(\Delta_{+\mathbf{y}}^{\bar{l}} f\right)(\mathbf{x})}{\mathbf{y}^{1+\bar{\alpha}}} d \mathbf{y}+\int_{\mathbb{R}_{-}^{n}} \frac{\left(\Delta_{-\mathbf{y}}^{\bar{l}} f\right)(\mathbf{x})}{(-\mathbf{y})^{1+\bar{\alpha}}} d \mathbf{y}\right] \\
& =\frac{\chi_{\bar{l}}(\bar{\alpha})}{d_{n, \bar{l}}(\bar{\alpha})}\left[\left(\mathbf{D}_{+}^{\alpha} f\right)(\mathbf{x})+\left(\mathbf{D}_{-}^{\alpha} f\right)(\mathbf{x})\right]
\end{aligned}
$$

with $\bar{\alpha}=(n-1)+\alpha$ and $\bar{l}=(n-1)+l=\bar{\alpha}$. Eq. (47) may also be written, under the assumption $0 \leqslant \alpha \leqslant 1$ as (see [35], Eqs. (25) and (26)):

$$
\begin{aligned}
\left(\mathrm{D}^{\alpha} f\right)(\mathbf{x}) & =\frac{1}{d_{n, \bar{l}}(\bar{\alpha})} \int_{\mathbb{R}^{n}} \frac{f(\mathbf{y})-f(\mathbf{x})}{\|\mathbf{x}-\mathbf{y}\|^{n+\alpha}} d \xi \\
& =\frac{\chi_{\bar{l}}(\bar{\alpha})}{d_{n, \bar{l}}(\bar{\alpha})}\left[\left(\mathbf{D}_{+}^{\alpha} f\right)(\mathbf{x})+\left(\mathbf{D}_{-}^{\alpha} f\right)(\mathbf{x})\right]
\end{aligned}
$$

that corresponds, in case of multivariable function fields $f(\mathbf{x})$, to a relation between the Riesz and the Marchaud differential operators analogous to that involving scalar variable functions reported in Eq. (41).

Fractional operators on multivariable fields will be used, in the following subsections, to model the fractional long-range thermal energy transfer in a $3 \mathrm{D}$ rigid conductor.

\subsection{The fractional-order Fourier transport equation}

Let us assume that the temperature field is due to a thermal pulse acting at $t=0$ in the entire topological space and let us assume that the distance-decaying function $g(\|\mathbf{x}-\mathbf{y}\|)$ involved in the long-range thermal transfer function $\chi_{n l}(\mathbf{x}, \mathbf{y}, t)$ belongs to the functional class of the power-laws as:

$g(\|\mathbf{x}-\mathbf{y}\|)=\frac{1}{d_{n, \bar{l}}(\bar{\alpha})} \frac{1}{\|\mathbf{x}-\mathbf{y}\|^{n+\alpha}}$

where $\alpha \in \mathbb{R}$ and $n \in \mathbb{N}$ represents the dimension of the topological space of the body, in our case $n=3$. As far as the decaying function is chosen in the class expressed in Eq. (49), and we assume a homogeneous conductor, that is $\varrho(\mathbf{x})=\varrho(\mathbf{y})=\varrho$, the internal energy balance reported in Eq. (1) is written in the form:

$\rho C_{V} \frac{\partial T}{\partial t}=-\nabla \cdot \mathbf{q}+\rho^{2} \kappa_{\alpha}\left(D_{\mathbf{x}}^{\alpha} T\right)+\rho r$

where $\mathrm{D}_{\mathbf{x}}^{\alpha} T$ is the fractional derivative of order $\alpha$ as in Eq. (48), defined as:

$\mathrm{D}_{\mathbf{x}}^{\alpha} T=\frac{1}{d_{n, l}(\bar{\alpha})} \int_{R^{3}} \frac{[T(\mathbf{y}, t)-T(\mathbf{x}, t)]}{\|\mathbf{x}-\mathbf{y}\|^{3+\alpha}} d V_{\mathbf{y}}$

when Fourier law for the classical local transfer of thermal energy is used, we obtain:

$\frac{\partial T}{\partial t}=\frac{\lambda}{\rho C_{V}} \nabla^{2} T+\frac{\kappa_{\alpha}}{C_{V}}\left(\mathrm{D}_{\mathbf{x}}^{\alpha} T\right)+r$

where $\lambda$ is the (local) heat conductivity while $\kappa_{\alpha}$ is a coefficient, introduced in Eq. (4) that characterize the strength of the longrange thermal energy transfer.

The field of temperature distribution ruled by Eq. (52) in a 1D unbounded rigid body is provided by the solution of the fractional differential equation as: $\frac{\partial T}{\partial t}=\frac{\lambda}{\rho C_{V}} \nabla^{2} T+\frac{\kappa_{\alpha}}{C_{V}}\left(\mathrm{D}_{x}^{\alpha} T\right)$

where we neglected the presence of localized thermal energy sources at location $x$ that for the 1D case must be supplemented by the relevant initial and boundary conditions that reads:

$T(x, 0)=T_{0}(x)$

$T(-\infty, t)=T(\infty, t)=0$

Mathematical description of the temperature distribution field in rigid body may be obtained, in integral form for any distributed heat sources $r(x, t)$ as we obtain the Greens' function for a concentrated temperature distribution $T_{0}(x)=\bar{T}_{0} \delta(x)$. In this context the solution of Eq. (53) is obtained resorting to spatial Fourier transform of both sides of Eq. (53) yielding a differential equation for the spatial distribution of the temperature field in Fourier space $\widehat{T}(k, t)$ as:

$\frac{\partial \widehat{T}}{\partial t}=-\frac{\left(\lambda k^{2}+\rho \kappa_{\alpha}\|k\|^{\alpha}\right)}{\rho C_{V}} T$

with initial condition expressed as $\widehat{T}(k, 0)=\bar{T}_{0}$. Solution of Eq. (55) yields the temperature distribution field evaluating the Fourier integral:

$$
\begin{aligned}
T(x, t) & =\frac{\bar{T}_{0}}{2 \pi} \int_{-\infty}^{+\infty} e^{i k x} \widehat{T}(k, t) d k \\
& =\frac{\bar{T}_{0}}{2 \pi} \int_{-\infty}^{+\infty} e^{i k x} \exp \left[-\frac{\left(\lambda k^{2}+\rho \kappa_{\alpha}\|k\|^{\alpha}\right)}{\rho C_{V}} t\right] d k
\end{aligned}
$$

\subsection{The fractional-order Cattaneo temperature equation}

The effects of the ballistic energy transport may be highlighted considering the local transport equation in terms of the Cattaneo relaxation time. In this case, under the assumption of the previous section in terms of the temperature distribution field, the governing equation of the temperature reads:

$$
\tau \ddot{T}(\mathbf{x}, t)+\left(\dot{T}(\mathbf{x}, t)-\frac{\kappa_{\alpha}\left(\mathbf{D}^{\alpha}[\tau \dot{T}(\mathbf{x}, t)-T(\mathbf{x}, t)]\right)}{C_{V}}\right)=\frac{\lambda \nabla^{2} T(\mathbf{x}, t)}{\rho C_{V}}+r+\dot{r}
$$

that represent the $3 \mathrm{D}$ extension of the temperature equation reported for the 1D case in [5]. Analytical solutions of the temperature equation in presence of Marchaud fractional derivatives may be also obtained with Fourier transform method.

In this section we will confine the analysis of the temperature distribution to a 1D unbounded domain, neglecting the presence of internal heat sources $r(x, t)=\dot{r}(x, t)=0$, ruled by the initial value problem:

$\tau \ddot{T}(x, t)+\left(\dot{T}-\frac{\kappa_{\alpha}}{C_{V}}\left(D^{\alpha}[\tau \dot{T}(x, t)-T(x, t)]\right)\right)=\frac{\lambda}{\rho C_{V}} \nabla^{2} T(x, t)$

with initial conditions $T(x, 0)=T_{0}(x) ; \dot{T}(x, 0)=0$ and the regularity conditions $T(-\infty, t)=0 ; T(\infty, t)=0$. The solution of Eq. (58) may be obtained with the aid of Fourier transform yielding

$$
\begin{aligned}
& \frac{d^{2} \widehat{T}(\kappa, t)}{d t^{2}}+2 A(\kappa) \frac{d \widehat{T}(\kappa, t)}{d t}+B(\kappa) \widehat{T}(\kappa, t)=0 \\
& \widehat{T}(k, 0)=\hat{T}_{0} ; \quad \dot{\widehat{T}}(k, 0)=0
\end{aligned}
$$

with coefficients in Eq. (59) defined as:

$A(\kappa)=\frac{1-\tau \Lambda_{\alpha}\|\kappa\|^{\alpha}}{2 \tau} ; \quad B(\kappa)=\frac{\lambda \kappa^{2}-\tau \Lambda_{\alpha}\|\kappa\|^{\alpha}}{\tau}$

and the fractional-order force-flux coefficient $\Lambda_{\alpha}$ reads: $\Lambda_{\alpha}=\kappa \cos (\alpha \pi / 2) / \varrho C_{V}$. The solution of Eq. (59) is provided as usual linear combination of exponential functions with coefficients 

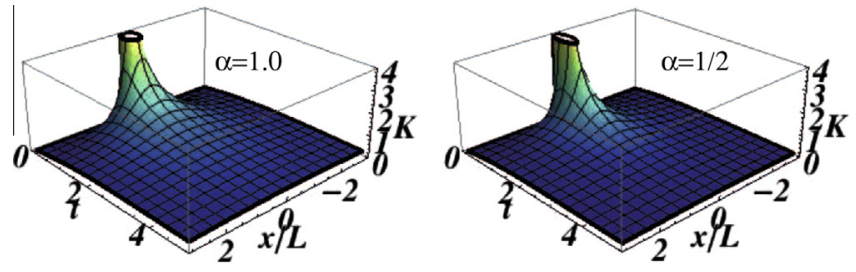

Fig. 1. Temperature distribution corresponding to long-range heat transport.

dependent on the initial conditions. In this regard, as we set, as in previous section, the initial temperature distribution as $T_{0}(x)=\bar{T}_{0} \delta(x)$, the solution reads:

$T(\mathrm{x}, t)=\frac{T_{0}}{2 \pi} \int_{-\infty}^{+\infty} \frac{r_{2}(\kappa) \exp \left[r_{1}(\kappa) t\right]-r_{1}(\kappa) \exp \left[r_{2}(\kappa) t\right]}{r_{1}(\kappa)-r_{2}(\kappa)} \exp [i \kappa x] d \kappa$

where $r_{1}(\kappa)$ and $r_{2}(\kappa)$ are the solution of the characteristic equation that are expressed in terms of the coefficients $A(\kappa)$ and $B(\kappa)$ as:

$r_{1}(\kappa)=-\left(A(\kappa)+\sqrt{A(\kappa)^{2}-B(\kappa)}\right) ; r_{2}(\kappa)=-\left(A(\kappa)-\sqrt{A(\kappa)^{2}-B(\kappa)}\right)$

\section{Numerical simulations of temperature distribution in 1D conductors}

In this section we will report some examples of temperature distributions obtained for the fractional-order temperature equations for the diffusive and the diffusive-ballistic thermal energy transport in 1D conductor. In case of a diffusive thermal energy transport, a closed-form expression for the temperature distribution in an unbounded domain due to an initial concentrated temperature pulse $T(0, x)=T_{0} \delta(x)$ has been obtained, under the assumption of vanishing local contributions $(\lambda=0)$ as combination of special functions for rational values of the parameter, respectively. In Fig. 1 we depicted the temperature field $T(x, t)$ for values of the differentiation order $\alpha=1, \frac{1}{2}$, respectively and with ratio $\frac{\kappa_{\alpha}}{C_{\mathrm{V}}}=1$ and $T_{0}=1$. It may be observed that, as $\alpha=1$ the Fourier integral in Eq. (56) reduces to a Cauchy-type temperature function as: $T(x, t)=\frac{2 t}{t^{2}+x^{2}}$ whereas, for $\alpha=1 / 2$ a linear combination of Fresnel integrals, namely, $S(x)$ and $C(x)$ represents the temperature distribution reported in Fig. 1 as:

$T(x, t)=\frac{\sqrt{\frac{\pi}{2}} t\left(\operatorname{Cos}\left[\frac{t^{2}}{4 x}\right]\left(1-2 C(x)\left[\frac{t}{\sqrt{2 \pi} \sqrt{\|x\|}]}\right)+\left(1-2 S(x)\left[\frac{t}{\sqrt{2 \pi} \sqrt{\|x\|}}\right]\right) \operatorname{Sin}\left[\frac{t^{2}}{4\|x\|}\right]\right)\right.}{\|x\|^{3 / 2}}$
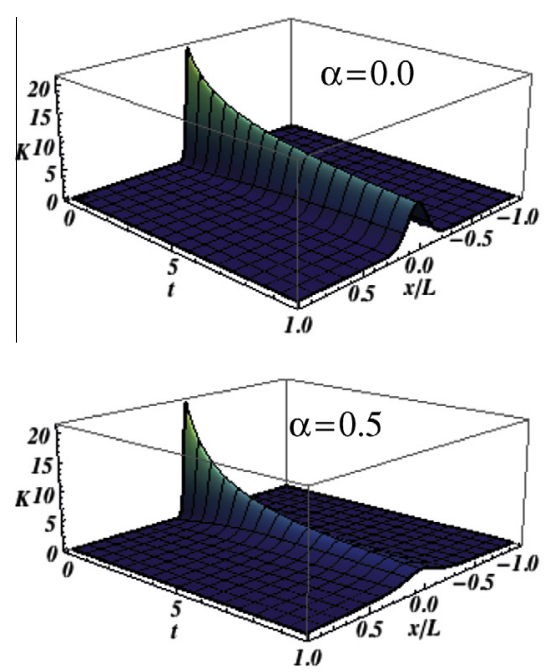

In passing we observe that closed-form solutions of the Fourier integral reported in Eq. (56) may be obtained as linear combination of special functions for any value of the differentiation order $\alpha \in \mathbb{Q}$ with $\mathbb{Q} \subset \mathbb{R}$ is the set of rational numbers by means of the series integration theorem.

A parametric study of the influence of the fractional-order derivative on the temperature distribution due to temperature pulse may be provided by the observation of Fig. 2 that reports the pure diffusive local transport, that is with $\tau=0$ and in this case Eq. (58) reverts to Eq. (53), for different values of the differentiation order $\alpha$.

The analysis has been conducted assuming a local diffusion coefficient $\frac{\lambda}{\rho C_{V}}=90$ and long-range transport coefficient $\frac{\kappa_{\alpha}}{C_{V}}$ and the initial temperature distribution has been assumed in the form:

$T_{0}(x)=\frac{\bar{T}_{0}}{\sqrt{2 \pi l_{T}}} \exp \left[-\left(\frac{x}{l_{T}}\right)^{2}\right]$

with $\bar{T}_{0}=100 \mathrm{~K}$ and the temperature spread parameter $l_{T}=\sqrt{200}$. The observation of Fig. 2 shows that as far as long-range thermal energy transfer is involved in Eq. (58) than a faster diffusion of the temperature pulse is observed with respect to the classical heat diffusion obtained as $\alpha=0$.

The presence of time lag $\tau=0.3 \mathrm{~s}$ in thermal energy transport induces the propagation of a decaying thermal wave that is strongly influenced by the fractional-order transport involved by long-range terms. The effect of the fractional differentiation order in the temperature distribution is reported in Fig. 3 that shows the presence of a thermal wave propagating in the $1 \mathrm{D}$ domain with a faster decay induced by the long-range diffusive transport. The presence of the faster decay observed for values of the differentiation order $\alpha \neq 0$ is in complete agreement with the presence of a long-range thermal energy transport, that does not contain any time lag in the constitutive relation in Eq. (4). Indeed, the longrange thermal energy transport depends on the relative temperatures only and as $\alpha \neq 0$ at any time instant lesser and lesser thermal energy remain concentrated in the solid domain perturbed by the thermal wave passage.

The effects induced by the time lag $\tau$ in the temperature distribution of the local and non-local type is showed in Fig. 4 reporting the temperatures of a $1 \mathrm{D}$ domain for different values of the time $\operatorname{lag}(\tau=0.05 \mathrm{~s} ; \tau=0.5 \mathrm{~s} ; \tau=1.0 \mathrm{~s} ; \tau=2.0 \mathrm{~s}$ ) and differentiation order $\alpha=1 / 2$ showing that, as time lag increases slower and slower thermal wave propagation may be observed (pure diffusion).
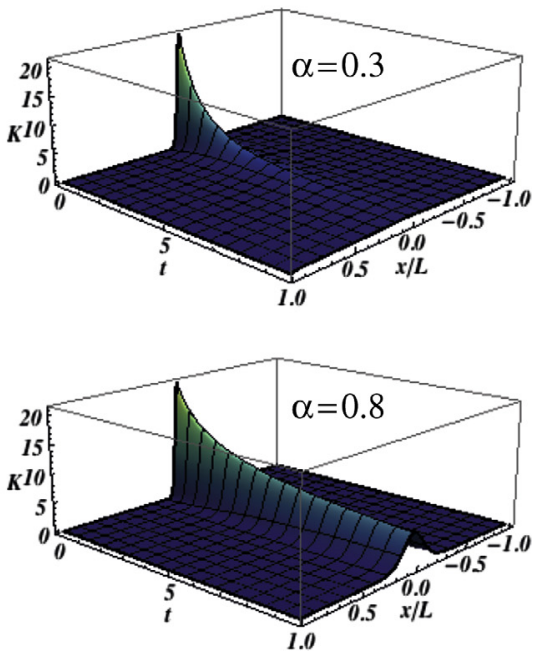

Fig. 2. Temperature distribution for Fourier-type transport equation. 

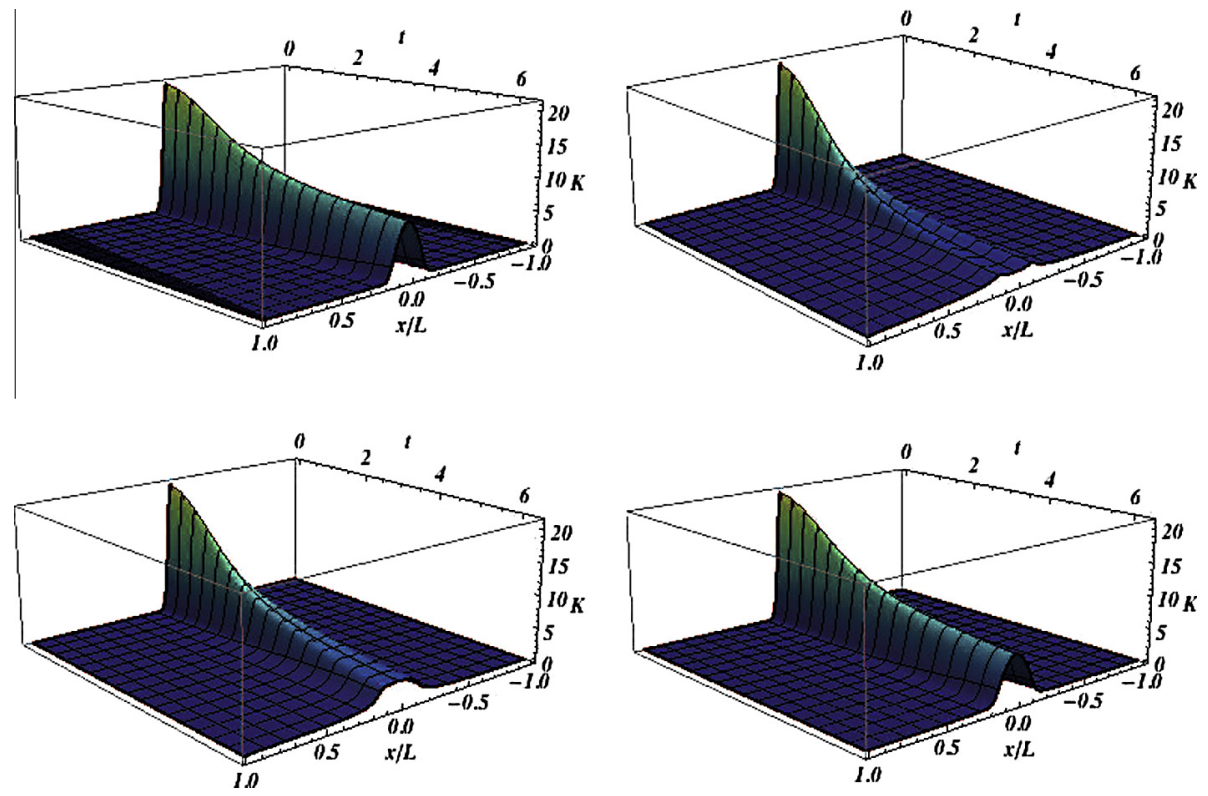

Fig. 3. Temperature distribution for Cattaneo-type transport equation.
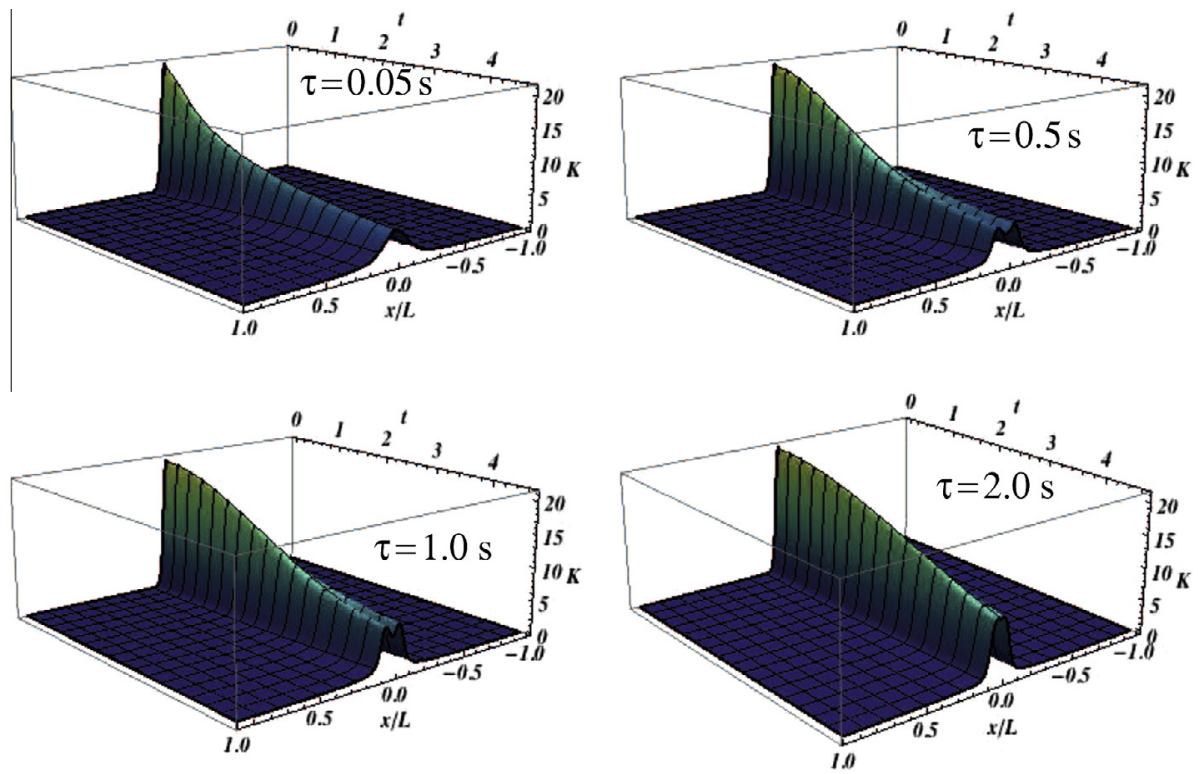

Fig. 4. Temperature distribution for Cattaneo-type transport equation varying the time lag.

Indeed, values of $\tau=0$ corresponds to unbounded speed of thermal waves propagation and, therefore, small values of the time lag involves a diffusion predominant thermal energy transport. In this case the effect of the fractional-order derivatives involved in the long-range energy transport is similar to the effect involved in pure diffusion as it may be observed by direct comparison of Fig. 4 with Fig. 2. On the other hand, as the value of the time lag increases, a smaller thermal waves may be observed and, in this case, the presence of the fractional-order terms yields a faster decay of the thermal waves since it contributes to diffuse thermal energy from the regions perturbed by the thermal waves and the colder ones that are outside the characteristic region involved by the local thermal energy propagation.

\section{Conclusions}

Thermal energy transfer in rigid heat conductor at nano-scales and/or for high frequency processes have been recently modeled by suitable extension of Fourier transport equation in terms of real-order (fractional) derivatives. Such extension relies on the long-tail properties of power-law kernels that are recently used to describe the slow spatial and temporal decay of temperatures observed at mesoscale of complex heterogeneous materials. Indeed at these scale, the presence of the material structure influences the thermal energy transport and the use of non-local thermodynamics has became very common introducing the scale effect as additional contributions to the transport equations. 
In this paper we framed the model in a more general context assessing the thermodynamic restrictions of the conductors' functional parameter class that may ensure a positive entropy rate for any thermodynamical process. Indeed, thermal energy transfer has been modeled as superposition of two contributions that coexhist at the considered observation scale: (i) A phonon collision/ballistic model of thermal energy transfer that is described by means of Fourier/Cattaneo transport equation and (ii) a phononic smallscale heat transport accounting for the long-range thermal energy transfer proportional to the relative temperature among interacting locations, to the product of interacting masses and to A proper, material-type decaying function. Proper restrictions on the functional class of the distance-decaying function has been reported in the paper showing that any thermal transfer decaying function that is strictly positive in the whole conductors' domain is eligible in Terms of second principle of thermodynamics.

As we assume that the decaying function belongs to the functional class of power-laws of the interacting distances then a fractional-order heat equation with Marchaud-type fractional derivatives of order $\alpha \in[0,1]$ In unbounded domains. As, instead, bounded domains are considered, then only integral terms of Marchaud fractional operator must be retained in the model since no thermal energy exchange among the interior of The body and the exterior space surrounding it is directly involved. This aspect is a peculiarity of the proposed model of long-range thermal energy transfer that prevent for the ill-conditioning of non-homogeneous Dirichlet and Neumann boundary conditions always encountered in integral non-local approaches.

Some numerical applications involving the temperature distributions and evolution in 1D domains for the Cattaneo and the Fourier local thermal energy transport have been provided in the paper to highlight the contribution of the non-local terms in the temperature wave propagation of Cattaneo model.

\section{Acknowledgments}

Maria Stella Mongioví acknowledges University of Palermo for the Grant 2012-ATE-0106. Massimiliano Zingales is very grateful to the financial support provided by the PRIN2010-11 "Stability, Control and Reliability of Flexible Structures" with National Coordinator Prof. A. Luongo.

\section{References}

[1] C.A. Eringen, An unified theory of thermomechanical materials, Int. J. Eng. Sci. 4 (1966) 179-202.

[2] C.A. Eringen, Theory of nonlocal thermoelasticity, Int. J. Solids Struct. 12 (1974) 1063-1077.

[3] M.D. Paola, M. Zingales, Long-range cohesive interactions of non-local continuum faced by fractional calculus, Int. J. Solids Struct. 45 (2008) $5642-$ 5659.

[4] K. Jolley, S.P.A. Gills, Modeling transient heat conduction at multiple lenght and time scale: a coupled equilibrium molecular dynamics/continuum approach, in: P.R. Eds (Ed.), Proc. of the IUTAM Symp, Nanomaterials and Nanosysistems, Pergamon Press, London, 2008.

[5] G. Borino, M. DiPaola, M. Zingales, A non-local model of fractional heat conduction in rigid bodies, Eur. Phys. J. Spec. Top. 193 (2010) 173-184.
[6] M.G. Caputo, Vibrations of a thin viscoelastic layer with a dissipative memory, J. Acoust. Soc. Am. 56 (1974) 704-897.

[7] H.R. Metzler, A. Blumen, T.F. Nonnenmacher, Generalized viscoelastic models: their fractional equations with solutions, J. Phys. A Math. Gen. 28 (1995) 65676584.

[8] C. Friedrich, Mechanical stress relaxation in polymers: fractional integral model versus fractional differential model, J. Non-Newtonian Fluid Mech. 46 (1993) 307-314.

[9] H.R. Metzler, T.F. Nonnenmacher, Fractional relaxation processes and fractional rheological models for the description of a class of viscoelastic materials, Int. J. Plast. 19 (2003) 941-959.

[10] I. Podlubny, Fractional Differential Equations, Academic Press, New-York, 1998.

[11] F. Mainardi, Y. Luchko, G. Pagnini, The fundamental solution of the space-time fractional diffusion equation, Fract. Calc. Appl. Anal. 4 (2001) 153-192.

[12] V.E. Tarasov, G.M. Zaslavsky, Conservation laws and hamiltonian's equations for systems with long-range interaction and memory, Commun. Non-Linear Sci. Numer. Simul. 13 (2001) 1870-1878.

[13] V.E. Tarasov, Fractional integro-differential equations for electromagnetic waves in dielectric media, Theor. Math. Phys. 158 (2009) 153-159.

[14] M.D. Paola, M. Zingales, Exact mechanical models of fractional hereditary materials (fhm), J. Rheol. 58 (2012) 986-1004.

[15] R.S. Lakes, J.L. Katz, Dielectric relaxation in cortical bone, J. Appl. Phys. 48 (1977) 808-811.

[16] C.A. Eringen, A non-local linear elasticity theory, Int. J. Eng. Sci. 4 (1972) 179202.

[17] K.A. Lazopoulos, Non local continuum through fractional calculus, Mech. Res. Commun. 33 (2006) 753-757.

[18] M. DiPaola, G. Failla, M. Zingales, Physically-based approach to the mechanics of strong non-local linear elasticity theory, J. Elast. 33 (2009) 102-130.

[19] Y.Z. Povstenko, Fractional heat conduction equations and associated thermal stress, J. Therm. Stress 28 (2005) 83-102.

[20] Y.Z. Povstenko, Two-dimensional axisymmetric stresses exerted by instaneus pulses and sources of diffusion in an infinite space in case of time-fractional diffusion equation, Int. J. Solids Struct. 44 (2007) 2324-2348.

[21] Y.Z. Povstenko, Theory of thermoelasticity based on space-time fractional heat conduction equation, Phys. Scrip. T136 (2009) 014017.

[22] T. Dedeurwaerdere, J. Casas-Vàzquez, D. Jou, G. Lebon, Foundations and applications of a mesoscopic thermodynamic theory of fast phenomena, Phys. Rev. E 53 (1996) 498-506.

[23] D. Jou, J. Casas-Vàzquez, G. Lebon, M. Grmela, A phenomenological scaling approach for heat transport in nano-systems, Appl. Math. Lett. 18 (2005) 936967.

[24] A. Compte, D. Jou, Non-equilibrium thermodynamics and anomalous diffusion, J. Phys. A Math. Gen. 29 (1996) 4321-4327.

[25] M.S. Mongioví, On linear extended thermodynamics of a non-viscous fluid in presence of heat flux, J Non-Equilib. Thermodyn. 25 (2000) 31-47.

[26] D. Jou, L. Restuccia, Mesoscopic transport equations and contemporary thermodynamics: an introduction, Contemp. Phys. 52 (2005) 465-474.

[27] B. Straughan, Heat Waves: Applied Mathematical Sciences, vol. 177, Springer, New York, 2011.

[28] H.H. Sherief, A. El-Sayed, A. Abd-El-Latief, Fractional order theory of thermoelasticity, Int. J. Solids Struct. 47 (2010) 269-275.

[29] H.M. Youssef, Theory of fractional-order generalized thermoelasticity, J. Heat Transfer 47 (2010) 269-275.

[30] G. Lebon, D. Jou, J. Casas-Vàzquez, Extended Irreversible Thermodynamics, Springer, New York, 2005.

[31] D. Jou, J. Casas-Vàzquez, G. Lebon, Undestanding Non-Equilibrium Thermodynamics, Springer, New York, 2010.

[32] Y.M. Youssef, Theory of fractional-order generalized thermoelasticity, J. Heat Transfer 47 (2010) 269-275.

[33] D. Jou, G. Lebon, M. Mongioví, R.A. Peruzza, Entropy flux in non-equilibrium thermodynamics, Physica A 338 (2004) 445-457.

[34] C. Cattaneo, Sulla conduzione del calore, Atti del Seminario di Mat.Fis. UniversitÃ di Modena p. 3 (in italian).

[35] S.G. Samko, A.A. Kilbas, O.I. Marichev, Fractional Integrals and Derivatives, Gordon Breach, Amsterdam, 1989. 